\title{
Ultrahigh-resolution optical coherence elastography
}

\author{
Andrea Curatolo ${ }^{1}$, Martin Villiger ${ }^{2}$, Dirk Lorenser ${ }^{1}$, Philip Wijesinghe ${ }^{1}$, \\ Alexander Fritz ${ }^{1}$, Brendan F. KenNedY ${ }^{1}$, DAVID D. SAMPSON ${ }^{1,3}$ \\ ${ }^{1}$ Optical+Biomedical Engineering Laboratory, The University of Western Australia, 35 Stirling Highway, Perth, WA 6009, Australia \\ ${ }^{2}$ Wellman Center for Photomedicine, Harvard Medical School and Massachusetts General Hospital, 40 Blossom Street, Boston, Massachusetts \\ 02114, USA \\ ${ }^{3}$ Centre for Microscopy, Characterisation and Analysis, The University of Western Australia, 35 Stirling Highway, Perth, WA 6009, Australia \\ *Corresponding author: andrea.curatolo@uwa.edu.au
}

Received XX Month XXXX; revised XX Month, XXXX; accepted XX Month XXXX; posted XX Month XXXX (Doc. ID XXXXX); published XX Month XXXX

Visualizing stiffness within the local tissue environment at the cellular and sub-cellular level promises to provide insight into the genesis and progression of disease. In this paper, we propose ultrahigh-resolution optical coherence elastography, and demonstrate threedimensional imaging of local axial strain of tissues undergoing compressive loading. We combine optical coherence microscopy and phase-sensitive detection of local tissue displacement to produce strain elastograms with resolution $(x, y, z)$ of $2 \times 2 \times 15 \mu \mathrm{m}$. We demonstrate this performance on freshly excised mouse aorta and reveal the mechanical heterogeneity of vascular smooth muscle cells and elastin sheets, otherwise unresolved in a typical, lower resolution optical coherence elastography system. (C) 2015 Optical Society of America

OCIS codes: (110.4500) Optical coherence tomography; (140.3300) Laser beam shaping; (100.2980) Image enhancement; (170.6935) Tissue characterization.

http://dx.doi.org/10.1364/OL.99.099999

Disease often changes the mechanical properties of tissue, providing a source of contrast for diagnosis distinct to that provided by optical properties [1]. Physical palpation, the most commonly used diagnostic method based on mechanical properties, enables physicians to detect abnormalities, but is subjective and provides very coarse resolution. Elastography is a medical imaging technique based on the spatially resolved response of tissue to mechanical loading and can provide a map of mechanical properties. Elastography based on magnetic resonance imaging or ultrasound imaging [2] provides better resolution than manual palpation and is emerging as a clinical tool in the diagnosis liver fibrosis and breast cancer [3]. Optical coherence elastography (OCE) [4], a form of elastography based on optical coherence tomography (OCT), shows promise in visualizing mechanical contrast in tissue with resolution of tens of micrometers, enabling the probing of mechanical properties on a scale intermediate between that of cells and organs. However, disease changes the mechanical properties of tissue on even smaller cellular to sub-cellular length scales [5]. Indeed, the field of cell mechanics seeks to study the onset and development of disease on the cellular scale and to characterize the mechanical signatures of healthy and diseased cellular tissue constituents [6]. Ideally, such studies would include the ability to provide in situ images at cellular resolution in live tissues in their native environment.

Atomic force microscopy [7] can be used to probe the mechanical properties on this and still finer scales, but it has a small field of view (FOV), typically hundreds of $\mu \mathrm{m}^{2}$, providing measurements from the tissue surface only, and is not compatible with in vivo imaging.

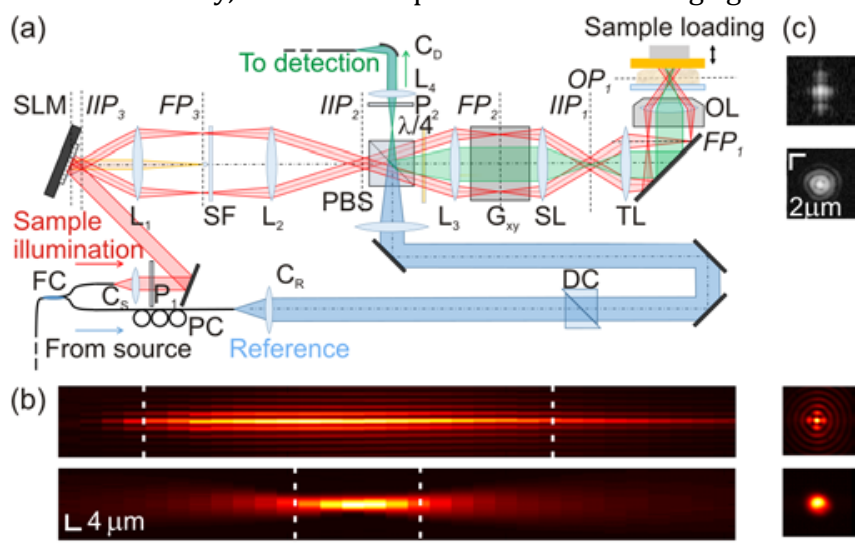

Fig. 1. UHROCE system. (a) Schematic of the sample and reference arms of the extended-focus Fourier-domain optical coherence microscopy (xf-FDOCM) system. C: collimator DC: dispersion compensation, $\mathrm{FC}$ : fiber coupler, $\mathrm{G}_{\mathrm{x} y \mathrm{y}}$ : galvanometer mirrors, $\mathrm{L}$ : lens, OL: objective lens, SF: spatial filter, SL: scan lens, SLM: spatial light modulator (angle exaggerated), P: polarizer, PBS: polarizing beam splitter, PC: polarization controller, TL: tube lens, $\lambda / 4$ : quarter-wave plate, IIP: intermediate image plane, FP: Fourier plane, $O P$ : object plane. (b) Measured irradiance profiles of the beams (Bessel - top, Gaussian bottom) around $O P_{1}$ in the illumination and detection paths along (left) and transverse to (right) the propagation direction. The white dashed lines bound the depth of field of both modes. (c) Measured xf-FDOCM point-spread function in focus (axial - top, transverse - bottom). 
(a)

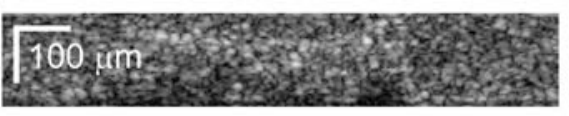

(b)

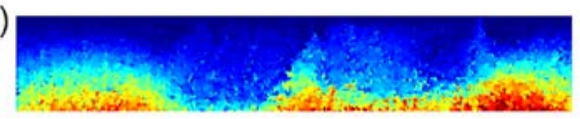

(c)

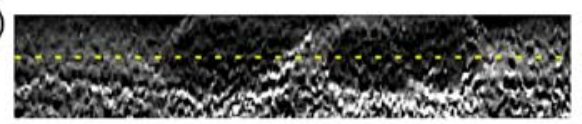

(d)

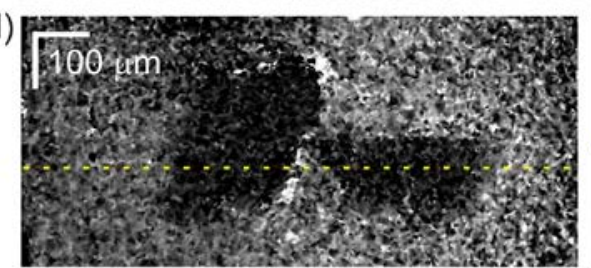

OCE
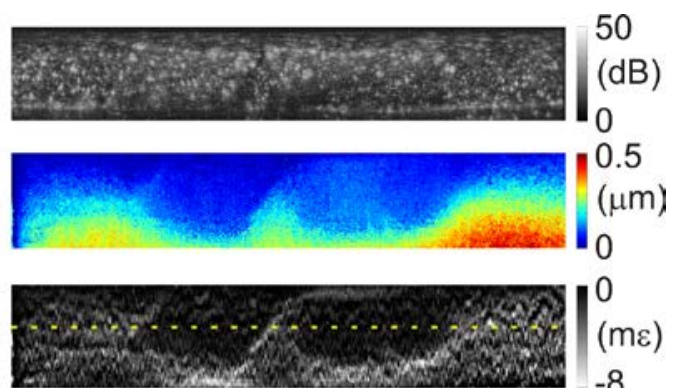

$-8$

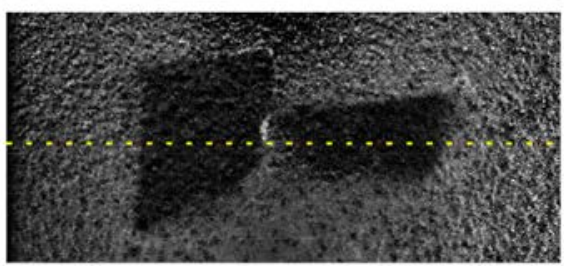

UHROCE

Fig. 2. OCT images and strain elastograms of an inclusion phantom taken with the two systems: OCE (left panels, OCT resolution: 7.8 axial x 11 $\mu \mathrm{m}$ transverse) and UHROCE (right, OCT resolution: 1.5 axial x $1.6 \mu \mathrm{m}$ transverse). (a) OCT B-scans. (b) B-scan displacement maps. (c) B-scan strain elastograms. (d) En-face strain elastograms. The location of the images in (c) and (d), respectively, is indicated by yellow dotted lines.

Optical coherence microscopy (OCM), in both its Fourier-domain and full-field implementations [8], is a high-resolution version of OCT providing spatially isotropic resolution below $2 \mu \mathrm{m}$. Elastography techniques based on OCM have the ability to probe sub-surface mechanical contrast overcoming a main limitation of AFM $[9,10]$.

A limitation of elastography based on Fourier-domain OCM is the technique's small depth of field (DOF), imposed by the high numerical aperture required to achieve ultrahigh in-focus resolution [8]. On the other hand, the ability to acquire a depth scan of the sample structure, with a single spectral acquisition, is an attractive feature that enables phase-sensitive displacement measurements. The dynamic focusing ability of full-field OCM overcomes the DOF limitation, but at the expense of severely restricting accurate phase-sensitive displacement detection. To date, the employment of a digital volume correlation (DVC) method [11] on a rather large 3D sliding window has limited the strain resolution of full-field OCM.

In this paper, we seek to address the above shortcomings by achieving simultaneously high strain sensitivity and high spatial resolution. We do so via ultrahigh-resolution optical coherence elastography (UHROCE), which combines extended-focus Fourierdomain optical coherence microscopy (xf-FDOCM) [12], phasesensitive detection, and compressive mechanical loading [13]. Bessel beam illumination and Gaussian mode detection [14] maintain sub$2 \mu \mathrm{m}$ transverse resolution over a nearly $100 \mu \mathrm{m}$ DOF. Phase-sensitive detection enables nanometer-scale displacement sensitivity over a wide displacement dynamic range from less than $1 \mathrm{~nm}$ to $\sim 2 \mu \mathrm{m}$ [15]. We demonstrate UHROCE in three spatial dimensions on a structured silicone phantom and on freshly excised mouse aorta, comparing UHROCE images with co-located typical, lower resolution OCE images [13]. The comparison highlights the unprecedented strain resolution of UHROCE and its ability to visualize the distribution of features, such as $10 \mu \mathrm{m}$-thick elastin sheets in the tunica media of mouse aorta, otherwise unresolved in the lower resolution strain elastograms.

In the UHROCE system, broadband light from a supercontinuum source (SuperK Extreme EXR-1, NKT Photonics, Denmark) is spectrally shaped to produce a spectrum with central wavelength $\lambda_{0}=785 \mathrm{~nm}$ and $3 \mathrm{~dB}$ bandwidth of $200 \mathrm{~nm}$. The source output is coupled to one arm of a $2 \times 2$ broadband fiber coupler forming part of a Mach-Zehnder interferometer. The optical design of the sample arm employs pointspread function (PSF) engineering and beam scanning in a three-stage telescope system, depicted in Fig. 1(a). A Bessel beam (Fresnel number
$\mathrm{N}=10.5$ ) generated by a spatial light modulator (SLM) (Pluto NIR IIHR, Holoeye Photonics AG, Germany) provides a good trade-off between improved DOF and reduced peak intensity compared to an equal resolution Gaussian beam [16]. The Bessel beam is relayed from the intermediate image plane $3\left(\mathrm{IIP}_{3}\right)$ to the object plane $(\mathrm{OP})$ within the sample, with an effective demagnification of $\sim 21 \mathrm{x}$. The illumination path (effective numerical aperture, $\mathrm{NA}_{\text {eff }}=0.27$ ) and separate detection path $\left(\mathrm{NA}_{\mathrm{eff}}=0.16\right)$ result in a Bessel illumination DOF of 94 $\mu \mathrm{m}$ and a Gaussian detection DOF of $21 \mu \mathrm{m}$. Figure $1(\mathrm{~b})$ shows the axial plane and in-focus transverse plane illumination and detection irradiance profiles, acquired using a beam-profiling camera (SP620U, Ophir-Spiricon, USA). Figure 1(c) shows cross-sections of a PSF near focus, i.e., the OCM signal generated by imaging a phantom comprising red iron oxide $\left(\mathrm{Fe}_{2} \mathrm{O}_{3}\right) 300-800 \mathrm{~nm}$-sized particles (refractive index, $n \approx 3)$ embedded in polyurethane resin $(n=1.49)$ (National Physical Laboratory, UK). The measured axial and transverse resolutions are 1.5 and $1.6 \mu \mathrm{m}$, respectively.

Measurements of the phase stability were carried out to benchmark the displacement sensitivity of the xf-FDOCM system against an inhouse, common-path OCT system [13]. The best displacement sensitivity calculated between groups of four A-scans, at an A-scan frequency of $20 \mathrm{kHz}$, is $2.3 \mathrm{~nm}$. The best displacement sensitivity for the common-path OCT system, at an A-scan frequency of $10 \mathrm{kHz}$, is $0.34 \mathrm{~nm}$ [13]. Such comparatively large phase fluctuations in the xfFDOCM system are due to the relatively long path lengths employed in the Mach-Zehnder interferometer setup. A common-path configuration would reduce these fluctuations, but it could not be implemented due to the effectively "dark-field" design [14], necessary to reduce the otherwise saturating signal from the sample coverslip. Mechanical loading was introduced using a piezoelectric transducer [13] to compress the sample, from the opposite side to that imaged.

The signal processing chain involves calculating the phase difference between a pair of complex xf-FDOCM B-scans of the uncompressed (pre-loaded) and compressed sample acquired at the same $y$-location. Axial unwrapping of the resulting phase difference [13] allows for unambiguous calculation of the local displacement in the sample. Local strain was estimated from the displacement map following the weighted-least squares (WLS) method described in [15]. To reduce the WLS regression window on the measured axial displacement for a given strain sensitivity, i.e., to exploit the xf-FDOCM resolution improvement, additional steps in the acquisition and signal processing 
scheme were required. Phase averaging was performed to improve the displacement sensitivity [13]. At each transverse $x$-location, four Ascans were acquired for each of the uncompressed and compressed sample states, enabling the averaging of their respective phase differences, thereby providing increased displacement sensitivity with no loss of spatial resolution. Similarly, two pairs of uncompressedcompressed B-scans per $y$-location were acquired and the resulting colocated B-scans strain elastograms were averaged.

A structured tissue-mimicking phantom with well-determined optical and mechanical properties was fabricated using twocomponent, room-temperature vulcanizing silicone (Elastosil@, Wacker, Germany) [17]. It comprised two stiff inclusions separated laterally by less than $20 \mu \mathrm{m}$, in a $325 \mu \mathrm{m}$-thick soft bulk. The optical properties were controlled by adding titanium dioxide $\left(\mathrm{TiO}_{2}\right)$ nanoparticles (refractive index $n \approx 2.51$, average diameter $25 \mathrm{~nm}$ ) to the silicone. A scatterer volume ratio of 3:1 between the inclusion and the bulk provided optical contrast. A stiffness (Young's modulus) ratio of 3:1 between the inclusion and the bulk provided mechanical contrast. The Young's modulus of the inclusion was $150 \mathrm{kPa}$ and that of the bulk was $50 \mathrm{kPa}$. These values were measured for a preload of $4 \mathrm{kPa}$ applied to the bulk, corresponding to $10 \%$ strain.

We acquired UHROCE images of this phantom. Comparative images of the same phantom were taken with the lower resolution OCE system, with an OCT resolution of $7.8 \mu \mathrm{m}$ (axial, in air) by $11 \mu \mathrm{m}$

(a)

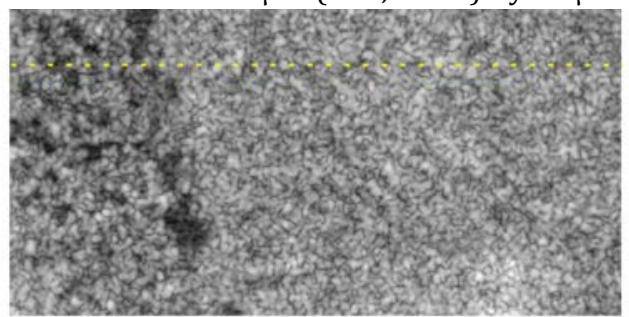

(b)

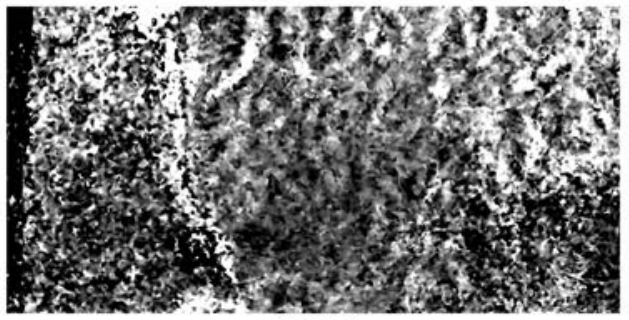

(c)

OCE
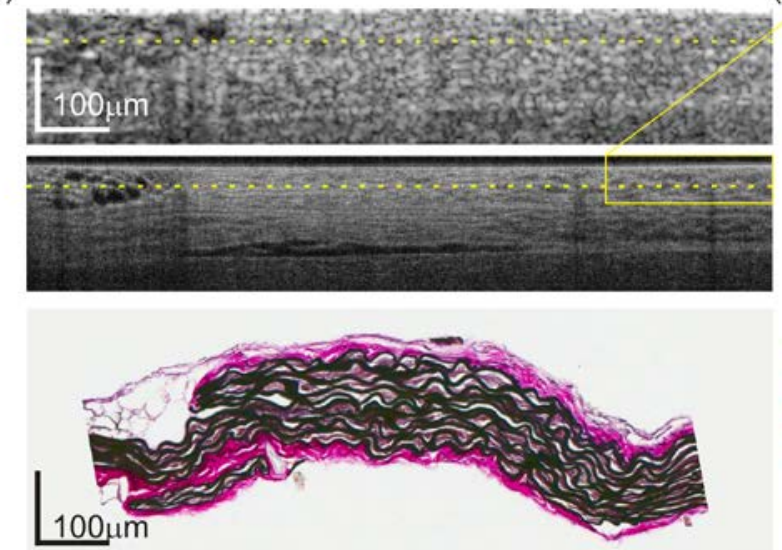

Fig. 3. OCT images and strain elastograms of a mouse aorta taken with the two systems: OCE and UHROCE, compared with histology. (a) en-face OCT images within the tunica media. (b) Corresponding en-face strain elastograms. (c) OCT B-scan images of the aorta cross-section (taken with OCE - top - and UHROCE system - middle), and a VVG-stained histology image (bottom panel) from a closely located but not corresponding section. (d) Speckle-averaged magnified portion (top inset) of the structural B-scan image in (c), corresponding B-scan strain elastogram (middle inset), and representative histology section (bottom inset) showing interleaved elastin sheets and smooth muscle cell layers. (transverse) [13]. The amount of phase averaging applied was similar to that used in the UHROCE system and the same axial fitting range window of $20 \mu \mathrm{m}$ ( $15 \mu \mathrm{m}$ FWHM equivalent) was used for strain estimation. For both systems, the additional bulk compression of the inclusion phantom applied during acquisition produced $\sim 0.3 \%$ strain.

The results of this comparison are presented in Fig. 2: for the OCE system at left; and for the UHROCE system at right. Figure 2(a) presents OCT B-scan sections of the phantom cutting through the two inclusions. The two inclusions are much better resolved in the xfFDOCM image, as expected, and clumps of $\mathrm{TiO}_{2}$ nanoparticles can be clearly observed. Figure 2(b) shows the displacement B-scans. The UHROCE displacement B-scan (at right) appears smoother over the chosen axial fitting length. This is related to the higher spatial frequency of the "granularity" in the image. This factor depends on the interplay between the noise sources in the displacement measurement and the axial pixel sampling density. Slightly lower OCT SNR, and higher strain- and displacement-induced decorrelation [18], due to the smaller xf-FDOCM average speckle size (i.e., the higher resolution at focus [19]), introduce more phase (difference) noise per pixel in the UHROCE displacement image. Nevertheless, the higher axial pixel sampling density within the given axial fitting length is the reason for the higher strain sensitivity attained with the UHROCE system, visible in the B-scan strain elastograms shown in Fig. 2(c). A better appreciation of the transverse resolution improvement can be gained
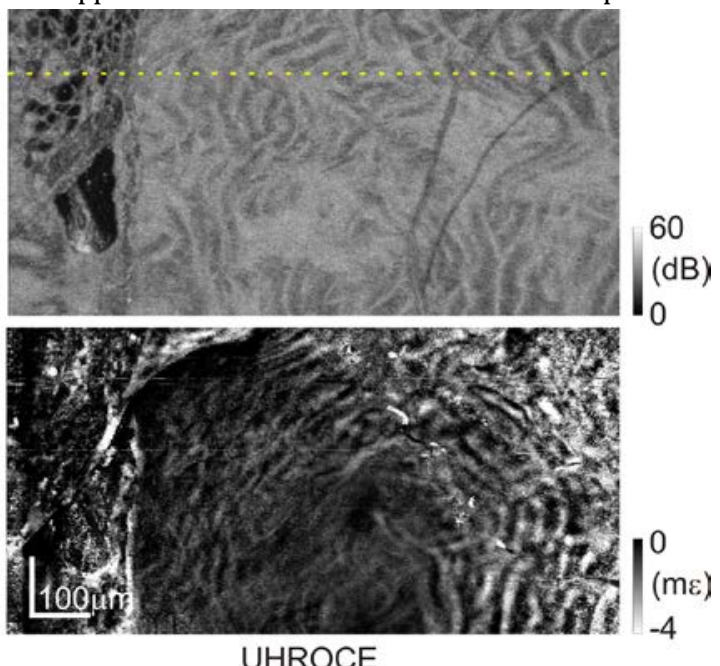

(d)
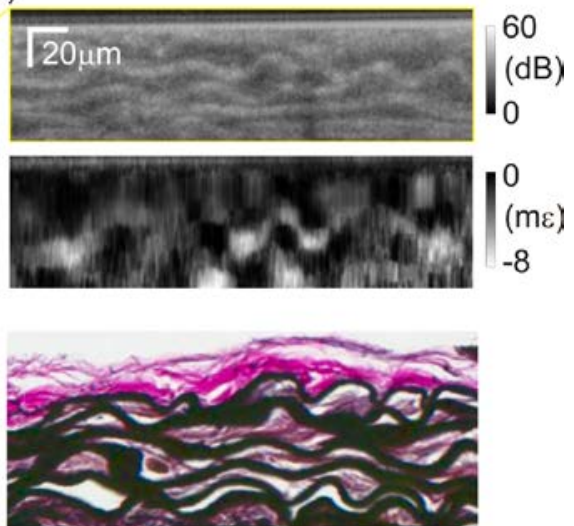

\section{)}


from the en-face strain elastograms in Fig. 2(d).

We also demonstrated the value of such resolution improvement by capturing strain elastograms of an ex vivo mouse aorta. A healthy mouse aorta wall is composed of three layers [20], progressing from the lumen outwards: tunica intima; tunica media; and tunica adventitia. The tunica intima is composed of one layer of endothelial cells followed by a layer of smooth muscle cells embedded in an extracellular matrix. The tunica media is made up of multiple smooth muscle layers interleaved with elastin sheets (lamellae) and fibers. The tunica adventitia is rich in collagen and is in contact with connective tissue, such as adipose cells. In our experiment, we imaged an excised aorta, compressed to flatten its lumen so that the tunica adventitia was lying flat against the compressing actuator and coverslip.

Figure 3 shows images of the mouse aorta acquired with both the OCE and the UHROCE systems, and compares them to histology of the same tissue. Figure 3(a) shows en-face OCT images taken mainly within the tunica media. The adipose tissue on the left aided in the colocation within a few hundred microns of images acquired on the two independent systems. The xf-FDOCM image (right) reveals sections of elastin sheets (lamellae), providing higher backscattering than the vascular smooth muscle, unresolved in the OCT image (left). The UHROCE strain elastogram (right) shows that the strain within the elastin sheets is low, whilst the smooth muscle layers in between them are subject to compressive strains of up to $0.4 \%$ in some areas. The improvement over the OCE image (left) is substantial, as features clearly resolved in the UHROCE image are only partially resolved at the lower resolution. Figures 3(c)-(d) show in detail the undulating structure of the elastin lamellae (black in the histology image with Verhoeff-Van Gieson (VVG) staining) sandwiching the smooth muscle, and resulting in a muscle layer of only a few cells in thickness experiencing high strain, as detected by the UHROCE system. (In Fig. 3(b), a phase unwrapping artifact in the UHROCE image has been removed by fusing an image at the same depth processed from the same data with different phase unwrapping parameters.)

The system and results presented here fall within the greater scope of utilizing OCM to measure tissue deformation. In this space, two groups $[9,10]$ have used magnetic fields to actuate exogenous agents (magnetic micro beads) dispersed in the tissue. They used Fourierdomain OCM systems for their inherent ability to detect nanometerrange tissue motion. The results were promising, however, tissue displacement was plotted only for specific points of interest in cell cultures, without providing an image of mechanical contrast. Another group [11] performed high-resolution compression OCE by using a piston to load the tissue and full-field OCM to detect the local displacement, from which they derived the strain tensor. They presented images of the axial strain experienced by compressed biological ex-vivo tissue, but, as mentioned above, the strain resolution suffered from a rather coarse DVC method of evaluating tissue displacement.

We have proposed and demonstrated compression UHROCE and compared it to a typical, lower resolution phase-sensitive, compression OCE system. The spatial resolution $(\mathrm{x}, \mathrm{y}, \mathrm{z})$ of the strain elastogram, at 2 $\times 2 \times 15 \mu \mathrm{m}$, is the highest reported to date in optical elastography. We have demonstrated the advance in terms of resolution by imaging an inclusion phantom and an ex vivo mouse aorta. The results show the ability of UHROCE to resolve mechanical heterogeneity at the micrometer scale, suggesting the method could be suitable for imaging of cell mechanics in situ in tissues [21, 22]. A challenge for stiffness quantification at the cellular level with the proposed technique is the uneven stress distribution within the flattened sample. The addition of methods of estimating local stress [23] would allow this technique to be made quantitative along the axial direction and produce elastograms representing tissue stiffness (Young's modulus), similar to those obtained with dynamic full-field OCE [21], but at a higher spatial resolution. Such a combination of resolution, depth of field, and quantitative mechanical properties would represent a unique combination in the study of mechanobiology.

We acknowledge Dr. Barry Doyle for providing and preparing the mouse aorta tissue sample; CELLCentral, at The University of Western Australia, for preparation of the histology slides and the facilities of the Australian Microscopy \& Microanalysis Research Facility at the Centre for Microscopy, Characterisation \& Analysis, The University of Western Australia, a facility funded by the University, State and Commonwealth Governments. A. C. was supported by an Australian Postgraduate Award, M. V. by a Gledden Visiting Fellowship, and P. W. by the William and Marlene Schrader Scholarship, all provided by The University of Western Australia. We acknowledge funding from the National Health \& Medical Research Council, the Australian Research Council and the National Breast Cancer Foundation.

\section{References}

1. G. Y. H. Lee, and C. T. Lim, Trends in Biotechnology 25, 111-118 (2007).

2. J. F. Greenleaf, M. Fatemi, and M. Insana, Annu. Rev. Biomed. Eng. 5, 5778 (2003)

3. W. A. Berg, D. O. Cosgrove, C. J. Doré, F. K. W. Schäfer, W. E. Svensson, R. J. Hooley, R. Ohlinger, E. B. Mendelson, C. Balu-Maestro, M. Locatelli, C. Tourasse, B. C. Cavanaugh, V. Juhan, A. T. Stavros, A. Tardivon, J. Gay, J.-P. Henry, and C. Cohen-Bacrie, Radiology 262, 435-449 (2012).

4. B. F. Kennedy, K. M. Kennedy, and D. D. Sampson, IEEE J. Sel. Topics Quantum Electron. 20, 272-288 (2014).

5. M. Plodinec, M. Loparic, C. A. Monnier, E. C. Obermann, R. ZanettiDallenbach, P. Oertle, J. T. Hyotyla, U. Aebi, M. Bentires-Alj, Y. H. LimRoderick, and C.-A. Schoenenberger, Nat. Nano. 7, 757-765 (2012).

6. D. T. Butcher, T. Alliston, and V. M. Weaver, Nat. Rev. Cancer 9, 108-122 (2009).

7. H.-J. Butt, B. Cappella, and M. Kappl, Surf. Sci. Rep. 59, 1-152 (2005).

8. A. F. Fercher, W. Drexler, C. K. Hitzenberger, and T. Lasser, Rep. Prog. Phys. 66, 239 (2003).

9. E. J. McDowell, A. K. Ellerbee, M. A. Choma, B. E. Applegate, and J. A. Izatt, J. Biomed. Opt. 12, 044008-044011 (2007).

10. V. Crecea, B. W. Graf, K. Taewoo, G. Popescu, and S. A. Boppart, IEEE J. Sel. Top.Quantum Electron. 20, 25-31 (2014).

11. A. Nahas, M. Bauer, S. Roux, and A. C. Boccara, Biomed. Opt. Express 4, 2138-2149 (2013).

12. R. A. Leitgeb, M. Villiger, A. H. Bachmann, L. Steinmann, and T. Lasser, Opt. Lett. 31, 2450-2452 (2006).

13. B. F. Kennedy, R. A. McLaughlin, K. M. Kennedy, L. Chin, A. Curatolo, A. Tien, B. Latham, C. M. Saunders, and D. D. Sampson, Biomed. Opt. Express 5, 2113-2124 (2014).

14. M. Villiger, C. Pache, and T. Lasser, Opt. Lett. 35, 3489-3491 (2010).

15. B. F. Kennedy, S. H. Koh, R. A. McLaughlin, K. M. Kennedy, P. R. T. Munro, and D. D. Sampson, Biomed. Opt. Express 3, 1865-1879 (2012).

16. D. Lorenser, C. Christian Singe, A. Curatolo, and D. D. Sampson, Opt. Lett. 39, 548-551 (2014).

17. G. Lamouche, B. F. Kennedy, K. M. Kennedy, C.-E. Bisaillon, A. Curatolo, G. Campbell, V. Pazos, and D. D. Sampson, Biomed. Opt. Express 3, 13811398 (2012).

18. L. Chin, A. Curatolo, B. F. Kennedy, B. J. Doyle, P. R. T. Munro, R. A. McLaughlin, and D. D. Sampson, Biomed. Opt. Express 5, 2913-2930 (2014). 19. A. Curatolo, B. F. Kennedy, D. Sampson, D., and T. R. Hillman, in Advanced Biophotonics (Taylor \& Francis, 2013), pp. 211-277.

20. T. Boulesteix, A. M. Pena, N. Pagès, G. Godeau, M. P. Sauviat, E. Beaurepaire, and M. C. Schanne-Klein, Cytometry Part A 69A, 20-26 (2006). 21. A. Nahas, M. Tanter, T. Nguyen, J. Chassot, M. Fink, and A. C. Boccara, J. Biomed. Opt. 18, 121514 (2013).

22. C.-E. Leroux, J. Palmier, A. C. Boccara, G. Cappello, and S. Monnier, New J. Phys. 17, 073035 (2015).

23. K. M. Kennedy, L. Chin, Robert A. McLaughlin, Bruce Latham, Christobel M. Saunders, D. D. Sampson, B. F. Kennedy, Sci. Rep. 5, 15538 (2015). 


\section{Full references}

1. G. Y. H. Lee, and C. T. Lim, "Biomechanics approaches to studying human diseases," Trends in Biotechnology 25, 111-118 (2007).

2. J. F. Greenleaf, M. Fatemi, and M. Insana, "Selected methods for imaging elastic properties of biological tissues," Annual Review of Biomedical Engineering 5, 57-78 (2003).

3. W. A. Berg, D. O. Cosgrove, C. J. Doré, F. K. W. Schäfer, W. E. Svensson, R. J. Hooley, R. Ohlinger, E. B. Mendelson, C. Balu-Maestro, M. Locatelli, C. Tourasse, B. C. Cavanaugh, V. Juhan, A. T. Stavros, A. Tardivon, J. Gay, J.-P. Henry, and C. Cohen-Bacrie, "Shear-wave Elastography Improves the Specificity of Breast US: The BE1 Multinational Study of 939 Masses," Radiology 262, 435-449 (2012).

4. B. F. Kennedy, K. M. Kennedy, and D. D. Sampson, "A review of optical coherence elastography: fundamentals, techniques and prospects," IEEE J. Sel. Topics Quantum Electron. 20, 272-288 (2014).

5. M. Plodinec, M. Loparic, C. A. Monnier, E. C. Obermann, R. ZanettiDallenbach, P. Oertle, J. T. Hyotyla, U. Aebi, M. Bentires-Alj, Y. H. LimRoderick, and C.-A. Schoenenberger, "The nanomechanical signature of breast cancer," Nature Nanotechnology 7, 757-765 (2012).

6. D. T. Butcher, T. Alliston, and V. M. Weaver, "A tense situation: forcing tumour progression," Nature Reviews Cancer 9, 108-122 (2009).

7. H.-J. Butt, B. Cappella, and M. Kappl, "Force measurements with the atomic force microscope: Technique, interpretation and applications," Surface Science Reports 59, 1-152 (2005).

8. A. F. Fercher, W. Drexler, C. K. Hitzenberger, and T. Lasser, "Optical coherence tomography - principles and applications," Reports on Progress in Physics 66, 239 (2003).

9. E. J. McDowell, A. K. Ellerbee, M. A. Choma, B. E. Applegate, and J. A. Izatt, "Spectral domain phase microscopy for local measurements of cytoskeletal rheology in single cells," Journal of Biomedical Optics 12, 044008-044011 (2007).

10. V. Crecea, B. W. Graf, K. Taewoo, G. Popescu, and S. A. Boppart, "High Resolution Phase-Sensitive Magnetomotive Optical Coherence Microscopy for Tracking Magnetic Microbeads and Cellular Mechanics," IEEE Journal of Selected Topics in Quantum Electronics 20, 25-31 (2014).

11. A. Nahas, M. Bauer, S. Roux, and A. C. Boccara, "3D static elastography at the micrometer scale using Full Field OCT," Biomedical Optics Express 4 2138-2149 (2013).

12. R. A. Leitgeb, M. Villiger, A. H. Bachmann, L. Steinmann, and T. Lasser, "Extended focus depth for Fourier domain optical coherence microscopy," Optics Letters 31, 2450-2452 (2006).

13. B. F. Kennedy, R. A. McLaughlin, K. M. Kennedy, L. Chin, A. Curatolo, A. Tien, B. Latham, C. M. Saunders, and D. D. Sampson, "Optical coherence micro-elastography: mechanical-contrast imaging of tissue microstructure," Biomedical Optics Express 5, 2113-2124 (2014).

14. M. Villiger, C. Pache, and T. Lasser, "Dark-field optical coherence microscopy," Optics Letters 35, 3489-3491 (2010).

15. B. F. Kennedy, S. H. Koh, R. A. McLaughlin, K. M. Kennedy, P. R. T. Munro, and D. D. Sampson, "Strain estimation in phase-sensitive optical coherence elastography," Biomedical Optics Express 3, 1865-1879 (2012).

16. D. Lorenser, C. Christian Singe, A. Curatolo, and D. D. Sampson, "Energy-efficient low-Fresnel-number Bessel beams and their application in optical coherence tomography," Optics Letters 39, 548551 (2014).

17. G. Lamouche, B. F. Kennedy, K. M. Kennedy, C.-E. Bisaillon, A. Curatolo, G. Campbell, V. Pazos, and D. D. Sampson, "Review of tissue simulating phantoms with controllable optical, mechanical and structural properties for use in optical coherence tomography," Biomedical Optics Express 3, 13811398 (2012).

18. L. Chin, A. Curatolo, B. F. Kennedy, B. J. Doyle, P. R. T. Munro, R. A. McLaughlin, and D. D. Sampson, "Analysis of image formation in optical coherence elastography using a multiphysics approach," Biomedical Optics Express 5, 2913-2930 (2014).

19. A. Curatolo, B. F. Kennedy, D. Sampson, D., and T. R. Hillman, "Speckle in Optical Coherence Tomography," in Advanced Biophotonics (Taylor \& Francis, 2013), pp. 211-277.

20. T. Boulesteix, A. M. Pena, N. Pagès, G. Godeau, M. P. Sauviat, E. Beaurepaire, and M. C. Schanne-Klein, "Micrometer scale Ex Vivo multiphoton imaging of unstained arterial wall structure," Cytometry Part A 69A, 20-26 (2006).

21. A. Nahas, M. Tanter, T. Nguyen, J. Chassot, M. Fink, and A. C. Boccara, "From supersonic shear wave imaging to full-field optical coherence shear wave elastography," Journal of Biomedical Optics 18, 121514 (2013).

22. C.-E. Leroux, J. Palmier, A. C. Boccara, G. Cappello, and S. Monnier, "Elastography of multicellular aggregates submitted to osmomechanical stress," New Journal of Physics 17, 073035 (2015).

23. K. M. Kennedy, L. Chin, Robert A. McLaughlin, Bruce Latham, Christobel M. Saunders, D. D. Sampson, B. F. Kennedy, Quantitative microelastography: imaging of tissue elasticity using compression optical coherence elastography" Scientific Reports 5, 15538 (2015). 